\title{
FERIMENTO EM ZONA DE ZIEDLER POR ARMA BRANCA COM LESÃO CARDÍACA: RELATO DE CASO
}

\section{ARTIGO ORIGINAL}

AGUIAR, Gislanne dos Santos ${ }^{1}$

SANTOS, Victor Fróes Gomes ${ }^{2}$

RIBEIRO, Elton Gustavo Boralli ${ }^{3}$

LIMA, Naiara Lorrani Silva de ${ }^{4}$

RIBEIRO, Lucas Almeida ${ }^{5}$

SANTANA, Denys Costa ${ }^{6}$

${ }^{1}$ Acadêmica do $11^{\circ}$ período do curso de medicina pela Universidade Federal do Amapá (UNIFAP).

${ }^{2}$ Acadêmico do 9o período do curso de medicina pela Universidade Federal do Amapá (UNIFAP).

${ }^{3}$ Acadêmico do $11^{\circ}$ período do curso de medicina pela Universidade Federal do Amapá (UNIFAP).

${ }^{4}$ Acadêmico do 9o período do curso de medicina pela Universidade Federal do Amapá (UNIFAP).

${ }^{5}$ Acadêmico do $11^{\circ}$ período do curso de medicina pela Universidade Federal do Amapá (UNIFAP).

${ }^{6}$ Graduado em Medicina pela Universidade Federal do Maranhão, Cirurgião Geral pela Universidade Federal do Amapá. 
SEGUNDO, Prisco de Paiva Bezerra ${ }^{7}$

AGUIAR, Gislanne dos Santos. Et al. Ferimento em zona de ziedler por arma branca com lesão cardíaca: Relato de caso. Revista Científica Multidisciplinar Núcleo do Conhecimento. Ano 05, Ed. 11, Vol. 10, pp. 32-41. Novembro de 2020. ISSN: 2448-0959, Link de acesso: https://www.nucleodoconhecimento.com.br/saude/lesao-cardiaca

\section{RESUMO}

Em 2019, cerca de 1,2 milhões de internações relacionadas a morbidade por causas externas foram registradas. Assim, dentre esses casos, verificou-se internações de origem traumática onde, a incidência do trauma torácico foi classificada em terceiro lugar pela epidemiologia dos traumatismos, perdendo apenas para o trauma ortopédico e crânio encefálico. Neste contexto, o tipo de trauma torácico (TT) predominante é o fechado, com incidência que varia entre $56,2 \%$ e $97 \%$. Enquanto o TT penetrante está mais relacionado com lesões nas estruturas torácicas e na estrutura cardíaca. O trauma cardíaco tem como quadro clínico desde pacientes assintomáticos, até mesmo choque hipovolêmico refratário e tamponamento cardíaco. O número de óbitos pode chegar até $92 \%$ nas vítimas com lesão cardíaca. A prevalência de óbitos é mais relacionada com as lesões perfurantes e transfixantes e possui como fator causal de mortes a perda sanguínea e em seguida o tamponamento cardíaco. A Conduta do trauma torácico, inicialmente depende da estabilidade hemodinâmica do paciente, isto define se haverá a realização da Avaliação Focalizada com Sonografia para Trauma (FAST) e avaliação secundária ou se há indicação de uma toracotomia de emergência. Dito isso, esse artigo teve como objetivo apresentar um caso de trauma torácico, em paciente de 28 anos, do sexo masculino, que deu entrada na emergência do Hospital de Emergência Osvaldo Cruz, no estado Macapá. Apresentando também uma análise do caso com base na literatura. Assim, tratou-se

7 Orientador. Graduado em Medicina pela Universidade Federal do Pará, Cirurgião Geral em serviço credenciado pelo MEC, Cirurgião Cardiovascular pelo Instituto Dante Pazzanese de Cardiologia, São Paulo. 
de um estudo baseado na metodologia de um relato de caso descritivo. Observando como resultado, a importância de uma administração hospitalar adequada, com atendimentos e diagnósticos ágeis e precoces, contribuindo para a diminuição de riscos e complicações nos processos de tratamento.

Palavras-chave: Contusões miocárdicas, Traumatismos Torácicos, Tamponamento Cardíaco.

\section{INTRODUÇÃO}

No ano de 2019, houve cerca de 1,2 milhões de internações relacionadas a morbidade por causas externas, sendo a região norte do país responsável por apenas 8,68\% desse total (BRASIL, 2020b). Em 2018, as causas externas ocuparam a quarta posição no ranking de mortalidade Brasileira (BRASIL, 2020d).

Houve um total de 150.814 óbitos por causas externas, sendo $37 \%$ destas mortes causadas por agressões, enquanto acidentes de transportes e lesões autoprovocadas totalizaram pouco menos de 35\%. (BRASIL, 2020c).

A Classificação internacional de Doenças (CID) inclui como lesões ou morbidades por causas externas os acidentes em gerais, como os de transporte, lesões autoprovocadas, quedas, afogamentos, agressões físicas e traumas, negligência e maus tratos (BRASIL, 2020a).

O trauma consiste em uma lesão física adquirida por meio de ação violenta ou abrupta externa com potencial de gerar danos de variáveis extensões ao indivíduo (SBAIT, 2020).

No Brasil, o perfil do indivíduo vítima de trauma, tem como característica a maioria masculino, entre 20 e 29 anos. Além disso, as principais causas são as quedas, em seguida, o acidente automobilístico e em terceiro lugar os traumas por violência, como os por arma de fogo, arma branca e agressões (DA SILVA et al., 2017). 
A epidemiologia dos traumatismos demonstra como principais, em ordem decrescente: o trauma ortopédico, trauma crânio-encefálico, trauma torácico, trauma abdominal, trauma raquimedular e queimaduras. Dentre estes, o trauma torácico (TT) tem como lesões mais comuns o Pneumotórax, Hemopneumotórax e Hemotórax, enquanto o trauma cardíaco penetrante tem uma incidência de apenas 5\% (DA SILVA et al., 2017).

A fisiopatologia do TT tem como foco a hipóxia, hipercapnia e acidose. Sendo a hipóxia a oferta inadequada de oxigênio aos tecidos, no trauma grave a hipovolemia gera também a hipoperfusão. Vale ressaltar que, o próprio trauma torácico gera alterações de trocas gasosas pelo colapso pulmonar, ou por alterações da pressão intratorácica, como o tamponamento cardíaco. A hipercapnia é gerada pela ventilação inadequada, retendo o dióxido de carbono em níveis tóxicos. A acidose metabólica ocorre pela hipoperfusão tecidual resultando na hipoventilação (KALIL; FUSTER, 2016).

O tipo de trauma torácico predominante é fechado, com incidência que varia entre $56,2 \%$ e $97 \%$ nas literaturas, tendo como representantes o trauma automobilístico por motocicleta e quedas. Há também os TT penetrantes, nele há maior prevalência de ferimentos por arma branca, seguidos por arma de fogo. (ZANETTE; WALTRICK; MONTE, 2019).

O TT penetrante ou aberto está mais relacionado com lesões nas estruturas torácicas, no entanto, o trauma contuso também pode causar lesões na estrutura cardíaca, tal situação possui péssimo prognóstico, podendo atingir um índice de até $97 \%$ de mortalidade (LANAU et. al., 2007).

A suspeita de trauma cardíaco deve existir sempre que houver lesões sobre a chamada zona "perigosa" ou área de Ziedler (Figura 1), a qual é delimitada pelas linhas verticais paraesternal direita e pela linha axilar média esquerda, as linhas horizontais pela fúrcula esternal e rebordo costal das décimas costelas (KALIL; FUSTER, 2016). 
Figura 1. Zona de Ziedler.

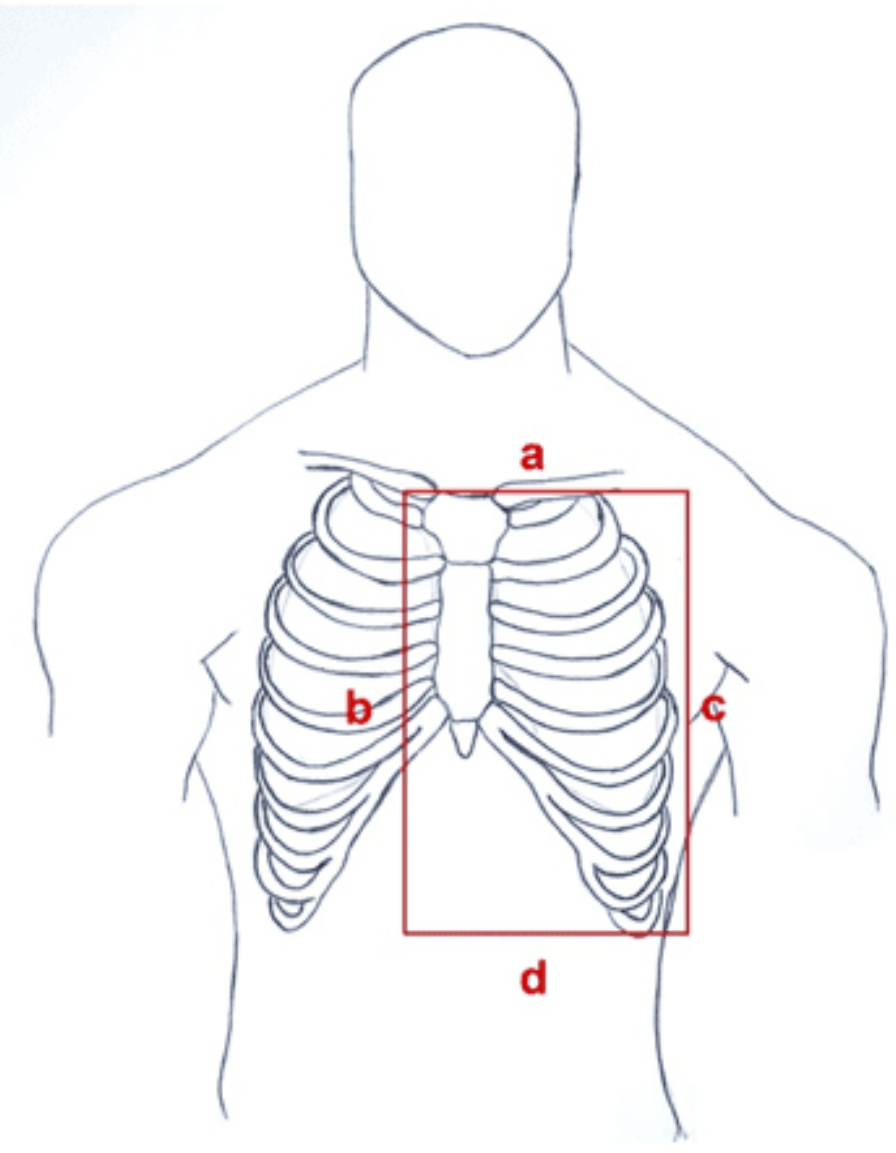

Fonte: Acervo Pessoal.

a) Linha horizontal à fúrcula esternal,

b) Linha paraesternal direita,

c) Linha paraesternal esquerda,

d) Linha horizontal do rebordo da décima costela.

A câmara cardíaca acometida com maior frequência é o ventrículo direito (VD), devido a sua posição anatômica (SOUZA; SANTOS; PEREIRA, 2013).

O quadro clínico do trauma cardíaco contuso ou perfurante varia entre assintomáticos, em feridas tamponadas, até choque hipovolêmico refratário ou mesmo tamponamento 
cardíaco, nos ferimentos que tiveram o sangramento contido pelo saco pericárdico, também lesões valvares ou ruptura de vasos. Os achados clínicos mais frequentes são dor torácica importante, dispneia, taquicardia e insuficiência cardíaca. Assim como a clássica tríade de Beck do tamponamento cardíaco, que consiste em abafamento de bulhas, elevação da pressão venosa central e hipotensão (KALIL; FUSTER, 2016).

O tamponamento requer diagnóstico e tratamento imediatos. Pois leva a diminuição do débito cardíaco e da perfusão coronariana, que podem resultar em isquemia miocárdica e choque obstrutivo, gerando a hipoperfusão tecidual, que tem papel importante na fisiopatologia do trauma torácico (MELO et. al., 2019).

\section{RELATO DE CASO}

Indivíduo do sexo masculino, 28 anos, natural e procedente de Macapá deu entrada na emergência do Hospital de Emergência Osvaldo Cruz, em Macapá, trazido pelo SAMU, vítima de agressão e acometido por múltiplos ferimentos por arma branca. Apresentava como sinais vitais a frequência cardíaca de 130 bpm e a frequência respiratória de 22 ipm.

Ao exame físico, foram evidenciadas 3 perfurações, sendo uma na face anterior do ombro direito, outras duas em hemitórax esquerdo. Inicialmente, apresentou-se com respiração espontânea, em uso de colar cervical e prancha rígida, presença de sangramento ativo moderado em lesões, com leve confusão mental e verbalizando pouco, escala de coma de glasgow 14 . O paciente estava hipocorado $2+/ 4+$, com ausculta cardíaca e pulmonar sem alterações.

Durante o atendimento inicial foi realizado acesso periférico, utilizado O2 (15 I min) sob máscara, administrado ringer lactato $2000 \mathrm{ml} \mathrm{EV}$, assim como 01 unidade de concentrado de hemácias.

Após a conduta inicial de suporte, o paciente evolui com piora, apresentando quadro de hipotensão arterial, seguido de hipofonese de bulhas cardíacas e discreto 
ingurgitamento jugular. Desta, foi sugerida a hipótese diagnóstica de tamponamento cardíaco. Sendo então encaminhado ao centro cirúrgico para realização de toracotomia imediata.

Ao chegar à sala de cirurgia e após preparação de monitorização, foi administrada a sedação. Durante o procedimento de toracotomia anterolateral esquerda, identificouse a exploração uma lesão no ventrículo esquerdo (VE), sendo executada a mio cardiorrafia, assim como a revisão da hemostasia e, posteriormente, posicionamento do dreno de tórax (esquerdo) em selo d'água, para drenagem torácica fechada. Por fim, foi realizada a sutura por planos da musculatura, sutura da pele e finalização com curativo.

Ao fim do procedimento cirúrgico, o paciente foi encaminhado para cuidados intensivos com prescrição do esquema de manutenção de sinais clínicos, como dieta zero, hidratação venosa, analgesia, antimicrobianos - Ceftriaxona e Metronidazol, concentrado de hemácias tipo O negativo, medicação sintomática e hidrocortisona, foi prescrito também a curva glicêmica e programado o início da dieta enteral.

Paciente evoluiu com melhora satisfatória, fez uso do dreno de tórax com débito sanguinolento, o qual foi retirado no quinto dia após a colocação cirúrgica. Teve alta da unidade de terapia intensiva após 4 dias para a enfermaria.

$\mathrm{Na}$ enfermaria do hospital, evoluiu com melhora e sem intercorrências clínicas, dessa forma, teve sua alta hospitalar redigida 10 dias após o incidente em bom estado geral.

\section{DISCUSSÃO}

O trauma penetrante tem relevância na ocorrência de traumas cardíacos, analisando um grupo 138 vítimas de lesões cardíacas, percebeu-se que a relação de óbitos chegou a $92 \%$. Apenas $16,7 \%$ das vítimas chegaram a ser atendidas no hospital, pois o óbito na cena ocorreu em $86,2 \%$ dos casos. Os locais anatômicos mais lesionados no coração foram: o ventrículo esquerdo (VE) nas lesões únicas, e nas lesões múltiplas, foram o VE e o ventrículo direito. A prevalência de óbitos estava relacionada 
a lesões perfurantes e transfixantes, e possuem como fator causal das mortes a perda sanguínea e em seguida o tamponamento cardíaco (ARAÚJO et al., 2018).

Os fatores que determinam a sobrevivência da vítima numa lesão cardíaca traumática e envolve o mecanismo da lesão, localização, lesões associadas, lesão coronariana, tamponamento cardíaco, cuidados no transporte pré-hospitalar, necessidade de toracotomia de resgate e experiência da equipe (GABRIELLI et al., 2007).

Para melhorar o atendimento, há uma sistematização de atendimento a todo paciente vítima de trauma, o Suporte de Trauma Avançado (ATLS), obra do Comitê do Trauma do Colégio Americano de Cirurgiões, que tem como objetivo organizar o atendimento inicial e sistematizá-lo, para que haja menor índice de mortes e sequelas nas vítimas de politraumas. O sistema segue o mnemônico ABCDE que orienta a ordem de avaliação e diagnóstico (OLIVEIRA, 2014).

O Atendimento inicial padronizado prioriza a proteção das vias aéreas, em seguida a respiração e suporte circulatório. A hipotensão no contexto do politrauma deve ser considerada devido a hemorragia, não disfunção cardíaca, a priori. Nas situações de lesões cardíacas penetrantes, a intervenção cirúrgica imediata é a prioridade. Todos os pacientes com suspeita de trauma cardíaco fechado, com ruptura cardíaca, lesão coronária ou lesão valvar devem ser atendidos por um cirurgião cardiotorácico de emergência (ALVARADO et al., 2016).

A Conduta do trauma torácico, inicialmente depende da estabilidade hemodinâmica do paciente, no caso de estabilidade, é sugerida a realização do FAST, se positivo é indicação de tratamento cirúrgico. Caso o FAST seja negativo é necessária uma avaliação secundária, se houver sugestão de pneumotórax há indicação de drenagem torácica. Caso o paciente esteja instável hemodinamicamente há indicação de uma toracotomia de emergência (KALIL; FUSTER, 2016).

A abordagem terapêutica ou cirúrgica é realizada de acordo com a complicação diagnosticada. Sendo elas tamponamento cardíaco, contusão miocárdica, ruptura 
cardíaca, síndrome coronariana aguda, arritmias e Lesão aórtica (ALVARADO et al., 2016).

No manejo de complicações, especificamente, do tamponamento cardíaco diagnosticado clinicamente pela tríade de Beck. O manejo deve focar na drenagem do sangue presente no saco pericárdico, realizando a pericardiocentese guiada por ultrassonografia, por exemplo. No manejo da ruptura cardíaca traumática, há associação de alta mortalidade, entre cerca de 60 a 100\% (MARIA et al.,2007).

\section{CONCLUSÃO}

Os ferimentos oriundos de origem traumática na área de Ziedler possuem risco de complicações, morbidade e mortalidade altas, no entanto, caso haja o manejo hospitalar adequado, de modo que, haja execução sistemática do Suporte Avançado de Vida no Trauma (ATLS) e conduta médica e cirúrgica imediatas, há uma melhora considerável do prognóstico dos pacientes. Ademais, é necessário promover o atendimento e diagnóstico precoces, desta forma, as medidas terapêuticas possam ser implementadas imediatamente, assim como diminuir o risco de complicações do tratamento e melhorar as chances de sobrevida.

\section{REFERÊNCIAS}

ALVARADO, C.; et. al. Trauma cardíaco cerrado. Revista Colombiana de Cardiologia, v. 23, $\quad$ n. $1, \quad$ p. $49-58$, jan. 2016. http://dx.doi.org/10.1016/j.rccar.2015.07.010.

ARAÚJO, A. O. D.; et. al. Trauma cardíaco fatal na cidade de Manaus/AM, Brasil. Revista do Colégio Brasileiro de Cirurgiões, [s.l.], v. 45, n. 4, p. 1-8, 4 out. 2018. FapUNIFESP (SciELO). http://dx.doi.org/10.1590/0100-6991e-20181888.

BRASIL. Ministério da Saúde. Capítulo XX Causas externas de morbidade e de mortalidade (V01-Y98): CID-10. Disponível em: http://www2.datasus.gov.br/cid10/V2008/WebHelp/v01_y98.htm. Acesso em 15/04/2020a. 
BRASIL. Ministério da Saúde. Informações de Saúde: Epidemiológicas e Morbidade. Morbidade Hospitalar do SUS por causas externas - Por Local de $\begin{array}{lllll}\text { Internação } & - & \text { Brasil. } & \text { Disponível }\end{array}$ http:/tabnet.datasus.gov.br/cgi/deftohtm.exe?sih/cnv/fiuf.de Acesso em 15/04/2020b.

BRASIL. Ministério da Saúde. Informações de Saúde: Estatísticas Vitais. Óbitos por causas externas - Brasil. Disponível em http://tabnet.datasus.gov.br/cgi/deftohtm.exe?sim/cnv/ext10uf.def. Acesso em 15/04/2020c.

BRASIL. Ministério da Saúde. Informações de Saúde: Mortalidade Geral. Mortalidade - $\quad$ Brasil. $\quad$ Disponível em http://tabnet.datasus.gov.br/cgi/deftohtm.exe?sim/cnv/obt10uf.def. Acesso em 15/04/2020d.

DA SILVA, L. A. P.; et. al. Análise retrospectiva da prevalência e do perfil epidemiológico dos pacientes vítimas de trauma em um hospital secundário. Revista de Medicina, v. 96, n. 4, p. 245-253, 22 dez. 2017.

GABRIELLI, N. M.; et. al. Herida penetrante cardíaca. Cuadernos de Cirugía, [s.I.], v. 21, n. 1, p. $75-83,2007$. Sistema de Bibliotecas UACH. http://dx.doi.org/10.4206/cuad.cir.2007.v21n1-11.

KALIL, R. F.; FUSTER, V. Medicina cardiovascular: reduzindo o impacto das doenças / editores; editor associado Cícero Piva de Albuquerque. São Paulo, p. 17791991: Editora Atheneu, 2016.

LANAU, M. I. V. I. V.; et. al. Trauma cardíaco penetrante: factores pronósticos. Revista colombiana de cirugía, v. 22, n. 3, p. 148-156, 2007.

MARIA, E.; et. al. Right atrial free wall rupture after blunt chest trauma. Journal Of Cardiovascular Medicine, [s.I.], v. 8, n. 11, p. 946-949, nov. 2007. Ovid 
Technologies

(Wolters

Kluwer

Health).

http://dx.doi.org/10.2459/jcm.0b013e328013f8af.

MELO, A. L. A. V.; et. al. Ferimento por arma branca em zona de ziedler com lesão cardíaca: relato de caso: Relato de caso. Prevenção e Promoção de Saúde 6, [s.I.], p. 95-102, 18 dez. 2019. Atena Editora. http://dx.doi.org/10.22533/at.ed.38019181214.

OLIVEIRA, T. N. S. Trauma: Atendimento Inicial no Intra-Hospitalar. 2014. 22 f. Monografia (Especialização) - Curso de Enfermagem, Enfermagem, Universidade Federal de Santa Catarina, Florianópolis, 2014. Disponível em: https://repositorio.ufsc.br/bitstream/handle/123456789/173454/TELMA\%20NEVES\% 20DA\%20SILVA\%20OLIVEIRA\%20-\%20EMG\%20-

\%20TCC.PDF?sequence=1\&isAllowed=y. Acesso em 15/04/2020.

SBAIT. Sociedade Brasileira de Atendimento Integrado ao Traumatizado (SBAIT). O que é trauma? Disponível em: http://www.sbait.org.br/trauma.php. Acesso em $15 / 04 / 2020$

SOUZA, V. S.; SANTOS, A. C.; PEREIRA, L. V. Perfil clínico-epidemiológico de vítimas de traumatismo torácico submetidas a tratamento cirúrgico em um hospital de referência. Scientia Médica, Porto Alegre, v. 23, n. 2, p. 96-101, mar. 2013.

ZANETTE, G. Z.; WALTRICK, R. S.; MONTE, M. B. Perfil epidemiológico do trauma torácico em um hospital referência da Foz do Rio Itajaí. Revista do Colégio Brasileiro de Cirurgiões, [s.I.], v. 46, n. 2, p. 1-13, 2019. FapUNIFESP (SciELO). http://dx.doi.org/10.1590/0100-6991e-20192121.

Enviado: Outubro, 2020.

Aprovado: Novembro, 2020. 